\title{
CLINICAL PERSPECTIVE
}

\section{Missed Opportunities: The Case for Inpatient Lung Cancer Screening Joe Johnstone ${ }^{1}$, Andrea Covey ${ }^{2}$}

${ }^{1}$ University of Kansas Medical Center, Kansas City KS

${ }^{2}$ Kansas City Veterans Administration Hospital, Kansas City MO

Corresponding author: Joe Johnstone, University of Kansas Medical Center, 3901 Rainbow Boulevard, Kansas City KS, 66160 (jjohnstone@kumc.edu)

Received: March 12, 2021. Revised: March 27, 2021. Accepted: March 29, 2021. Published: March 31, 2021.

Am j Hosp Med 2021 March; 5(1):2021. DOI: https://doi.org/10.24150/ajhm/2021.003

Keywords: hospitalist, lung neoplasm, early detection of cancer, COVID-19, quality improvement

Over the past twenty years, the hospitalist field has grown from an uncommon metropolitan profession to a ubiquitous, broad specialty focused on the rapidly evolving field of inpatient medicine ${ }^{1}$. Hospitalists are no longer limited to acute medical conditions; the hospitalist in 2021 has an ever-broadening scope of practice and is increasingly seen as an expert in transitions of care, quality improvement, and high value medicine $^{2}$. Their role in public health is increasingly recognized by their work in infection control, antibiotic stewardship, addiction medicine, and smoking cessation to name a few. Hospitalists can continue their innovation and improve care by recommending and performing lung cancer screening (LCS). The coronavirus pandemic advanced the field of telehealth significantly and bundling of in-person services is the natural progression of this trend.
A divide between primary care and hospitalists was created by silo-ing medical care as well as by the advent of electronic medical records (EMR). Most inpatient settings now have EMR yet outpatient access remains variable. Many have tried bridging this with some hospitalists now reminding patients upon discharge to take care of their annual health screenings such as mammograms and colonoscopies. In surveys of hospitalized women due for breast cancer screening, nine out of ten thought it was important they discuss cancer screening with their hospitalist and a majority were willing to undergo inpatient screening ${ }^{3}$. A pilot program at an academic medical center demonstrated viability by harnessing the EMR to identify and automatically notify hospitalist of eligible women ${ }^{4}$. Seventeen of twenty-one patients were successfully screened without increasing length of stay. 
For a third of patients this was their first mammogram or were overdue by greater than four years. National data shows that lung cancer screening has been significantly underutilized prior to $\mathrm{Covid}^{5}$, and recent studies have confirmed that the pandemic has adversely affected routine screenings for breast and colon cancer ${ }^{6}$. The hospitalists' routine use of EMRs makes recommending screening for lung cancer on discharge a significant improvement in the standard of care. Innovative ideas such as using time during a non-pulmonary hospitalization to perform lung cancer screening would significantly increase adherence to national screening recommendations.

The National Lung Screening Trial (NLST) changed lung cancer from a grim diagnosis with a short life expectancy to a condition that can be screened for and often cured by identifying the first effective means of screening with low dose CTs (LDCT) ${ }^{7}$. Prior to this there were failed attempts at using chest x-rays and sputum cytology, all with no meaningful effect on mortality. Annual low dose CT surveillance produced a $20 \%$ decrease in lung cancer deaths and a $6 \%$ all-cause mortality benefit. The NELSON trial $^{8}$ confirmed significant benefits to LCS and the United States Preventive Services Task Force recently expanding lung cancer screening recommendations to current and former smokers aged 50-80 with a minimum 20 pack/year smoking history and life expectancy greater than five years. Raw application of NLST mortality benefits would avoid twelve thousand lung cancer deaths annually, however a 2019 study found in a population of over four thousand eligible patients, only fourteen percent had undergone screening9. With this nation's 36 million annual hospitalizations, the large population in need, and the noninvasive, rapid nature of LDCT, hospitalizations are an underutilized setting to provide lung cancer screening.

Modern technology and division of labor make recommending lung cancer screening well within the scope of a busy hospitalist. Eligibility criteria is straightforward for the clinician and screening algorithms implemented in EMRs are now quite sophisticated in excluding patients with ongoing malignancies or other contraindications. The required Medicare pre-counseling is reimbursed and may be performed by a nurse specialist. Following this the patient may undergo screening at any time.

The aspirational goal is a dedicated coordinator utilizing standardized tools to identify patients so that a quick, low radiation CT is performed at an opportune moment during the hospitalization. This would greatly reduce the time to first scan and capture patients who were referred but ultimately never underwent initial screening. A Veterans Administration study found that the presence of a nodule on initial screening was predictive of adherence at year two and three, indicating that undergoing this first scan is critical $^{10}$. The shift to virtual healthcare in response to the coronavirus pandemic creates a need for inpatient LCS. A decreased proportion of in-person healthcare visits necessitates bundling of services to respect 
our patients time and infection risk-tolerance. A non-pulmonary hospitalization may be a patient's best or only opportunity to undergo screening. Inpatient LDCT alleviates the need for a pre-screening counseling office visit and a trip to the outpatient radiology center. The hospitalist's increasing focus on quality improvement makes them a natural leader in implementing such a project at their institution.

The prevalence of the hospitalist profession has coincided with the development of lung cancer screening programs. This life saving intervention has led to early detection and treatment of many malignancies, but there are millions of eligible Americans who have yet to be reached. Innovative approaches such as screening patients for needed preventative care while hospitalized may bridge the gap between outpatient and inpatient medicine, and with the current pandemic, inpatient hospitalizations may be a crucial opportunity to offer an LDCT safely. With the aforementioned skills, unique place in our healthcare system, and the broad population served, hospitalists can improve patients' health by recommending lung cancer screening at hospital discharge and performing LDCTs during non-pulmonary hospitalizations.

Notes

Potential conflicts of interest: The author reports no conflicts of interest in this work.

\section{References}

1. Watcher RM, Goldmen L. Zero to 50,000 The 20th Anniversary of the Hospitalist. N Engl J Med 2016; 375:1009-1011.

2. Kisuule F, Minter-Jordan M, Zenilman J, Wright S. Expanding the roles of hospitalist physicians to include public health. J Hosp Med 2007 Mar;2(2):93-101.

3. Waseem $\mathrm{k}$, et al. Breast cancer screening preferences among hospitalized women. J Womens Health. Epub 2013 Jun 14.

4. Hwang A, et al. Targeted Inpatient Screening Mammogram Program to Reduce Disparities in Breast Cancer Screening Rates. Ann Fam Med. Nov-Dec 2014;12(6):556-8.

5. Zahnd W, Eberth J. Lung Cancer Screening Utilization: A Behavioral Risk Factor Surveillance System Analysis. Am J Prev Med. 2021 Jan-Feb; 19(1): 83.

6. Kaufman H, Chen Z, Niles J. Changes in the Number of US Patients With Newly Identified Cancer Before and During the Coronavirus Disease 2019 (COVID-19) Pandemic. JAMA Netw Open. 2020 Aug; 3(8): e2017267

7. The National Lung Screening Trial Research Team. Reduced Lung-Cancer Mortality with Low-Dose Computed Tomographic Screening. N Engl J Med 2011; 365:395-409

8. Koning H, Aalst C, Jong P, et al. Reduced Lung-Cancer Mortality with Volume CT Screening in a Randomized Trial. N Engl J Med. 2020 Feb 6;382(6):503-513. doi: 0.1056/NEJMoa1911793. Epub 2020 Jan 29.

9. Zahnd W, Eberth J. Lung Cancer Screening Utilization: A Behavioral Risk Factor Surveillance System Analysis. Am J Prev Med. 2019 Aug;57(2):250-255.

10. Tanner NT, Brasher PB, Wojciechowski B, et al. Screening Adherence in the Veterans Administration Lung Cancer Screening Demonstration Project. Chest. 2020 Oct;158(4):1742-1752. 